\title{
Diagnóstico sobre las Buenas Prácticas Docentes en la Facultad de Contaduría y Administración y la Facultad de Derecho de la Universidad Autónoma de San Luis Potosí
}

\section{Diagnostic about the Good Teaching Practice in the Faculty of Accounting and Administration and the Faculty of Law of the Autonomous University of San Luis Potosí}

\author{
Diana Cecilia Rodríguez Ugalde* \\ Fernando Mendoza Saucedo \\ Juana María Méndez Pineda
}

Universidad Autónoma de San Luis Potosí, México

\begin{abstract}
Pocas investigaciones se han realizado en el nivel superior sobre las buenas prácticas docentes, no obstante, de su importancia en ese nivel educativo. El objetivo del estudio fue identificar las características de las buenas prácticas docentes de un grupo de profesores de la Universidad Autónoma de San Luis Potosí (UASLP) que laboran en la Facultad de Contaduría y Administración (FCA) y la Facultad de Derecho (FD), con la finalidad de que puedan aportar a proyectos de formación en buenas prácticas. La información se obtuvo a través de entrevistas realizadas a alumnos con discapacidad, alumnos con mejor promedio y profesores. Para el análisis de los datos se utilizó el método de categorización de datos. Se obtuvieron características referentes a aspectos relacionales, valorales, didácticos y de identidad docente. Termina por plantear los datos obtenidos como guía para un futuro proceso de formación docente en buenas prácticas dentro de la FCA.
\end{abstract}

Descriptores: Enseñanza superior; Formación de docentes; Práctica pedagógica; Profesores; Universidad.

Few investigations have been carried out at the higher level on good teaching practices, despite their importance at that educational level. The objective of the study was to identify the characteristics of good teaching practices of a group of professors of the Autonomous University of San Luis Potosí (UASLP) who work in the Faculty of Accounting and Administration (FCA) and the Faculty of Law (FD), in order that they can contribute to training projects in good practices. The information was obtained through interviews with students with disabilities, students with better average and teachers. The data categorization method was used to analyze the data. Characteristics relating to relational, values, didactic and teacher identity aspects were obtained. It ends by presenting the data obtained as a guide for a future process of teacher training in good practices within the FCA.

Keywords: Higher education; Teachers; Teacher training; Teaching practice; University.

\footnotetext{
*Contacto: psic.diana.rdz.ugalde@hotmail.com 


\section{Introducción}

Los jóvenes que se vieron beneficiados en México por las políticas y acciones de integración o inclusión en la educación básica se encuentran en estos momentos en la posibilidad de acceder a la educación superior. Sin embargo, en este nivel pueden enfrentar barreras culturales, políticas o prácticas que les impidan acceder a la participación y el aprendizaje (Méndez y Mendoza, 2007). Con el fin de favorecer la inclusión educativa de todos los alumnos en la UASLP, el presente artículo surge de un trabajo de tesis de Maestría que tuvo como objetivo el desarrollo de buenas prácticas docentes del profesorado de la FCA a partir de una propuesta de formación docente basada en el método de investigación-acción (Rodríguez Ugalde, 2014)².

Aquí se muestra la primera fase del proyecto, que tuvo como objetivo identificar las características de las buenas prácticas docentes que llevan a cabo varios profesores y profesoras pertenecientes a la FD y la FCA. Es preciso decir que existen pocos trabajos dirigidos al desarrollo de buenas prácticas docentes en educación superior o que versen sobre el tema de educación inclusiva en ese nivel educativo. En este sentido, el trabajo que aquí se presenta forma parte de las acciones iniciales para incursionar en la inclusión dentro de las instancias pertenecientes a la UASLP.

\section{Revisión de la literatura}

En las últimas décadas las políticas neoliberales han propiciado una complicada forma de interactuar (García, 2004). Se habla de globalización, interconexiones, comunicación masiva, transnacionalización, dentro de un discurso que plantea un crecimiento y beneficios para todos: la "época de la prosperidad". Sin embargo, propicia paradójicamente el aislamiento, la enajenación del otro, el individualismo egoísta, la competencia, la discriminación, el rechazo, la segregación, la desconexión, la desigualdad (García, 2004; Ordine, 2013).

Existen empresas que dicen cuál es el estilo de vida deseable y cuál el desdeñable, así como las características personales deseables y desdeñables (Lloret, 1994; Ordine, 2013). El meollo de tales manifestaciones acoge en el centro el temor a la concepción del otro como diferente, así como el temor de concebirse a uno mismo como diferente (Contreras, 2002; Lloret, 1994). Al ser cuestionados por la diferencia (ésta surge en la relación) se manifiesta el riesgo a perder la certeza, y el comportamiento tiende a declinar hacia la asimilación o la segregación del otro; homogenizar al otro a lo que se considera conveniente o aprobable, violando con ello su integridad, su particularidad; o rechazarlo por la no homogenización (Gómez, 2008). Un nuevo tipo de imperialismo.

En este punto puede cuestionarse ¿qué se hace en las universidades? Lamentablemente en muchas, se perpetúa la segregación, la discriminación, la indiferencia a la diferencia, o la asimiliación. En la educación existe una gran tendencia a la homogenización que se manifiesta en el currículo, en las expectativas de los directivos, profesores, padres y hasta de los mismos estudiantes, en las prácticas de los docentes dentro del aula, en la

${ }^{1}$ La Tesis a la que se hace referencia, se tituló "Formación del profesorado en prácticas inclusivas en la FCA de la UASLP", autoría de Diana Cecilia Rodríguez Ugalde, se realizó en el marco de la Maestría en Psicología con énfasis en Educación y Sociedad, perteneciente a la Universidad Autónoma de San Luis Potosí. Se extiende el agradecimiento al Consejo Nacional de Ciencia y Tecnología por el apoyo otorgado a través de una beca nacional para el financiamiento del proyecto. 
interacción con los alumnos (Santos Guerra, 2006), y la universidad no escapa a ello. La educación universitaria se expande y sostiene de la idea de un sujeto específico, ideal, aún en el periodo actual de exigencia de flexibilidad. Bajo las políticas neoliberales, la universidad se apega a las mediciones cuantitativas y economicistas, en el marco de la visión empresarial, tras un discurso disfraz sobre la diversidad frente a un conjunto de acciones homogeneizantes (Ordine, 2013; Santos, 2015).

En la actualidad existen varios dilemas que se presentan en la visión de la educación inclusiva respecto a dónde es necesario incidir para permitir la inclusión, si tiene que observarse al alumno o si la mira debe de estar puesta en el contexto. Para calmar la incertidumbre sobre en dónde situar al ojo, se retoma aquí el enfoque de capacidades resumido por Nussbaum (2012), “[las capacidades] no son simples habilidades residentes en el interior de una persona, sino que incluyen también las libertades o las oportunidades creadas por la combinación entre esas facultades y el entorno político, social, económico" (p. 40). Por ello, corresponde atender e incidir en el entorno, debido a que en la medida en que el entorno permita el involucramiento íntegro de todas las personas, éstas se encontrarán en la posibilidad de desarrollar sus capacidades particulares al máximo.

Al hablar de inclusión dentro de la universidad, tendrá que cuidarse que no se quede como un término en el fondo del discurso, sino que implique la acción congruente. Evitar la inclusión excluyente, como cita Gentili (2001), al señalar cómo las instituciones educativas caminan con la bandera de educación de calidad para todos cuando aún prevalece la exclusión dentro de ellas. El camino hacia la inclusión en la universidad implica el involucramiento de todos, propiciar espacios donde puedan manifestarse las diferencias, que permitan el establecimiento de relaciones genuinas y no estereotipadas, y en donde se valore el aprendizaje particular de todos quienes la conforman.

En una propuesta realizada por Booth y Ainscow (2000) sobre el índice para la inclusión, dejan en manifiesto, aun cuando se oriente a la educación básica, tres esferas que requieren atención en cualquier institución educativa para lograr la educación inclusiva: a) cultura, b) políticas y c) prácticas. En este trabajo importa analizar las últimas en el nivel universitario. Por lo tanto, la práctica docente se entiende como aquello que realiza el maestro tanto dentro del aula como fuera de ella en orientación a su labor docente, en una bidimensionalidad con el pensamiento (Fierro, Fortoul y Rosas, 1999; Zabalza, 2012). Es decir, las acciones que van dirigidas a su quehacer con los alumnos que ocurren en un entorno social dentro de la institución educativa, la cual está inmersa en la sociedad.

Dicen Fierro, Fortoul y Rosas (1999) que la práctica docente comprende seis dimensiones: las acciones que realiza el docente en el aula -dimensión didáctica-; la institución que se inserta en la sociedad desde una postura específica con base en los aspectos políticoinstitucionales, administrativos y normativos -dimensión institucional-; las características particulares que definen al docente como persona -dimensión personal-; un conjunto de valores, creencias, actitudes y juicios propios que manifiesta en las relaciones con los agentes educativos, así como la normativa de la escuela, que se muestra en las reglas implícitas y explícitas -dimensión valoral-; el significado del docente sobre su tarea como agente educativo, las demandas sociales hacia su labor en relación con el contexto socio-histórico y político, y las condiciones geográficas y culturales -dimensión social-; la relación entre docentes y estudiantes, padres de familia, demás docentes, autoridades escolares, entre otros -dimensión interpersonal-. 
De modo que las buenas prácticas docentes son aquellas acciones dirigidas a mejorar el quehacer con el alumnado. Las buenas prácticas docentes variarán según el contexto social donde se esté situado, no existen buenas prácticas docentes universales. Además, involucran la inclusión educativa, en el sentido de que una buena práctica docente no es congruente con la exclusión (Zabalza, 2012). Así, podríamos referir como sinónimos la buena práctica docente y la práctica docente inclusiva.

Sobre los antecedentes, son pocos los trabajos que abordan las buenas prácticas docentes en el nivel de educación superior. Acorde con la orientación del estudio, se consideraron antecedentes pertinentes la propuesta de Zabalza (2012) sobre el análisis de las buenas prácticas docentes en nivel universitario, además de los trabajos realizados por Fernández, Maiques y Ábalos (2012) y Álvarez, Porta y Sarasa (2011). Zabalza (2012) propone identificar, analizar, representar y visibilizar las buenas prácticas docentes. Para ello, retoma a Larose y otros (citado en Zabalza, 2012) en la definición de tres vías de identificación de las buenas prácticas: a) el discurso sobre las prácticas, b) la respuesta a cuestionarios y pruebas, c) la observación directa o indirecta. El autor señala una amplia serie de pasos para analizar y visibilizar las buenas prácticas: a) revisión documental, b) selección de ejemplos de buenas prácticas, c) entrevistas a agentes de buenas prácticas, d) documentación de evidencias objetivas, e) triangulación, f) entrevistas a personas afectadas, g) identificación de características básicas, h) representación a través de mapas, diagramas o esquemas, i) visualizar núcleos sustantivos, j) definir patrones de generalización y desarrollar sus planteamientos.

Fernández, Maiques y Ábalos (2012) realizaron análisis de caso sobre dos profesores de la Universidad Politécnica de Valencia identificados como buenos docentes a través de las evaluaciones realizadas por los alumnos. La información se obtuvo por medio de entrevistas, grabación de clase y el estudio de recursos didácticos. En los resultados se encontró que dentro de su práctica estos profesores muestran una actitud reflexiva y crítica, compromiso ético con su labor docente y con la responsabilidad que conlleva trabajar con personas, realizan un trabajo sistemático y riguroso que integra la planeación, metodología y evaluación, utilizan estrategias que implican y retan a los alumnos, acercan su docencia a la realidad profesional, y se preocupan por la realidad de la docencia en la institución.

Por su parte, Álvarez, Porta y Sarasa (2011) realizaron su estudio sobre las buenas prácticas docentes en la formación del profesorado en la Universidad Nacional del Mar de Plata, para lo cual aplicaron un cuestionario a los alumnos avanzados con el fin de identificar a buenos docentes. A los profesores identificados les realizaron entrevistas sobre su experiencia como estudiantes y como docentes. Seleccionaron específicamente la información de un profesor de inglés y, a partir de la información tanto de los alumnos como del docente, determinaron categorías teóricas que conforman la buena práctica docente, estas fueron: la predisposición de ayudar y guiar, la promoción de un clima favorable al aprendizaje, flexibilidad, tolerancia, preocupación y responsabilidad, esfuerzo y compromiso, pasión e interés, desafían a los alumnos con la intención de motivarlos al alcance de objetivos y metas en el aprendizaje.

\subsection{Planteamiento del problema y justificación}

Históricamente, gran parte de la educación se ha edificado sobre políticas, prácticas y culturas excluyentes que se fundamentan en el paradigma del déficit (Ainscow, 2002; Booth y Ainscow, 2000; López Melero, 2001). Específicamente en la universidad, es 
posible percatarse que frecuentemente, desde el proceso de admisión, el diseño de evaluación segrega a aquellos alumnos que no cumplen con los estándares de la "normalidad", disminuyendo la posibilidad de que algunos jóvenes ingresen como estudiantes. Aun cuando los alumnos enfrentan tales barreras, varios se topan con que el currículo, las prácticas docentes y las instalaciones son excluyentes, lo cual puede afectar enormemente su paso por la educación superior, llegando algunos a desertar.

En la UASLP se han realizado esfuerzos para incidir en las prácticas que los profesores tienen dentro del aula en un intento por generar una educación inclusiva por parte del Centro de Investigación, Orientación y Apoyo a la Inclusión (CIOAI) (Amaro, Méndez y Mendoza, 2014; Camacho, 2013; López, Méndez y Mendoza, 2014; Vázquez, Méndez y Mendoza, 2015). ${ }^{2}$ A pesar de ello, aún se observan prácticas que no consideran a todos los alumnos en sus diferencias y que favorecen la exclusión (Amaro, Méndez y Mendoza, 2014). La FCA y la FD son de las facultades que tienen mayor número de estudiantes con discapacidad inscritos en sus aulas y es donde más trabajos de intervención se han realizado por el CIOAI para dirigirse hacia una educación inclusiva debido a la cultura y prácticas excluyentes que ocurren (Maya, 2016; Maya, Méndez y Mendoza, 2017; Vázquez, Méndez y Mendoza, 2015)³.

De este modo, el presente trabajo pretendió responder a la pregunta ¿cuáles son las características de las buenas prácticas docentes en la FCA y la FD que permiten el aprendizaje y la participación de todos los alumnos? El objetivo planteado fue conocer y analizar las características de las buenas prácticas docentes de los profesores de estas facultades, con el fin de que estas características sirvan en un futuro para el desarrollo de un proceso de formación del profesorado en buenas prácticas dentro de la FCA.

\section{Método}

La metodología desarrollada se basó en la comprensión compleja de las prácticas docentes. Lejos de optar por contar únicamente con la perspectiva del alumnado o del profesorado, acorde con la defensa de la inclusión como sentires, pensares y prácticas que involucran a todos y todas quienes conforman la comunidad educativa (Amaro, Méndez y Mendoza, 2014; López Melero, 2001; Maya, Méndez y Mendoza, 2017), se consideró conveniente incluir diferentes miradas y recurrir a la triangulación de datos de los y las participantes (Aguilar y Barroso, 2015). Con ello, como se mostrará, posteriormente se recurrió a la triangulación analítica de los datos, entre ellos, teoría y conjeturas de los autores (Aguilar y Barroso, 2015; Bertely, 2000).

\section{Diseño}

El presente trabajo es cualitativo de tipo exploratorio- descriptivo.

\footnotetext{
${ }^{2}$ El CIOAI es un centro anexo al Instituto de Ciencias Educativas de la UASLP.

${ }^{3}$ Cabe decir que la inclusión, y este proyecto en específico, no se orienta únicamente a estudiantes con discapacidad, sino que define a la diferencia como algo presente en todas las relaciones.
} 


\section{Participantes $^{t}$}

Se invitó como informantes clave a los alumnos con discapacidad que cursan las carreras ofrecidas por ambas facultades (tres alumnos de cada facultad; en la FCA dos hombres y una mujer, de primero, quinto y séptimo semestre; en la FD dos mujeres y un hombre, de quinto, séptimo y noveno semestre), debido a que se considera que son alumnos que presentan diferencias físicas evidentes ante las cuales los profesores (y otras personas) pueden mostrar actitudes de rechazo y segregación. Asimismo, se invitó a un alumno con mejor promedio de cada semestre en cada facultad, cinco mujeres y tres hombres (no se entrevistó a alumnos de primer semestre). Se consideró pertinente invitar a estos informantes debido a que el alto desempeño exige cierto tipo de prácticas docentes por parte del profesorado.

Por último, se invitó a seis profesores de tiempo completo; tres de cada Facultad. Cuatro tienen grado de maestría y dos de doctorado. Los profesores de la FCA fueron del género femenino, y los de la FD fueron una mujer y dos varones. Cuatro ocupan cargos administrativos además de los solicitados por el Programa para el Desarrollo del Profesorado (PRODEP) ${ }^{5}$, que van desde la coordinación del departamento de tutorías, la coordinación de posgrado y coordinación de carrera. Cinco de ellos tienen más de 15 años de experiencia en la docencia.

\section{Procedimiento/instrumentos}

A los estudiantes con discapacidad se les realizaron entrevistas no estructuradas a profundidad sobre los siguientes aspectos: experiencia a lo largo de su estancia en la Facultad, dificultades generales en su estancia, dificultades con los maestros de la Facultad respecto a las clases, características de una buena práctica docente (atención a la diversidad, método, actividades, exámenes, materiales, motivación, trabajo colaborativo, clima en el aula), características de una mala práctica docente, nombre de docentes que hacen buenas prácticas en su Facultad. Por su parte, a los estudiantes con mejor promedio se les realizaron entrevistas breves sobre las características de buenas prácticas docentes y la identificación de buenos docentes en sus facultades, a fin de cotejar con la información aportada por los alumnos con discapacidad.

Cuando se obtuvo la información señalada, se procedió a triangular los nombres de los buenos docentes identificados por los alumnos con discapacidad y los alumnos con mejor promedio. De esta manera se obtuvieron tres nombres de docentes en cada Facultad. Una vez identificados los buenos docentes se les invitó al proyecto. Se les realizó una entrevista no estructurada a profundidad sobre su práctica docente, respecto a lo que consideran que es una buena práctica docente, la formación docente, la atención a la diversidad, la experiencia de tener alumnos con diferencias evidentes como la discapacidad, lo que debe de considerar un maestro para atender a los alumnos al tomar en cuenta que todos son diferentes, dificultades en su trabajo docente.

\footnotetext{
${ }^{4}$ La cantidad de participantes fue pequeña debido a que la metodología elegida involucró procedimientos que requirieron tiempo y profundidad de indagación y análisis; además de que el trabajo que aquí se presenta formó la primera fase de un trabajo más amplio sobre formación en buenas prácticas docentes.

${ }^{5}$ El Programa para el Desarrollo del Profesorado es un programa de evaluación del profesorado que labora en la UASLP, donde se solicita el cumplimiento con cuatro actividades específicas: docencia, investigación, gestión y tutoría.
} 


\section{Análisis de datos}

Para la obtención de resultados se utilizó el método de categorización de datos propuesto por Bertely (2000). La autora propone un análisis compuesto de seis pasos: obtención de la información etnográfica; inscripción de la información; interpretación de la información por subrayados, inferencias factuales y conjeturas; estructuración de las categorías de análisis y patrones emergentes; triangulación de la información con la teoría de distintos autores, logrando con ello fundamentar o contrastar los hallazgos encontrados; escritura del texto interpretativo a partir de la triangulación de la teoría, de los sujetos participantes y las conjeturas del investigador. El análisis fue realizado y discutido por los tres autores del trabajo.

\section{Resultados 6}

A partir de la información analizada es posible notar las distintas características de las buenas prácticas docentes que señalan los alumnos y los profesores. Estas características se agruparon en cuatro de las seis dimensiones de la práctica docente que señalan Fierro, Fortoul y Rosas (1999), con lo cual se concretaron los datos como a continuación se muestra.

\subsection{Dimensión personal}

\subsubsection{Reflexionar sobre su propia docencia}

La reflexión sobre la práctica docente es la acción que permite a los profesores observarse, darse cuenta si su desempeño como docente ha sido adecuado, si hace falta realizar modificaciones, cambiar la metodología, el trato con los alumnos, si incluye a todos sus alumnos, si las actividades que plantea son las más indicadas, si está posibilitando la metacognición en sus alumnos, y el tipo de relaciones con sus alumnos.

Esta categoría fue mencionada únicamente por los docentes. Señalaron que es importante observar el desempeño de los alumnos y cuestionarse sobre lo que ellos como docentes realizan dentro del aula, analizar si se están cumpliendo los objetivos, si serían mejores otros métodos o actividades para que se logren los objetivos y para propiciar el involucramiento de los alumnos.

Pues esta materia de Administración que son las bases, los fundamentos, pues es mucha teoría, (se omite un segmento que no se considera relevante) entonces de repente tú decías es que estoy dando clases y te juro hasta yo misma me aburro, yo ya me aburrí, ya me aburrí, entonces fue mucho, el pensar en el cómo, cómo hacerle, qué hacer para que por ejemplo mi clase se les hiciera mucho más atractiva a los muchachos, que realmente ellos dijeran bueno, es que esto..., aun cuando es teoría, aun cuando es mucha teoría la que se está revisando, bueno pues algo me está dejando y es interesante.

(Docente AMG- FCA, p. 4)

Lo anterior coincide con lo mostrado por Porter y Brophy (1988) y Fernández (2002), al definir a la reflexión sobre la propia práctica docente como una característica de un docente incluyente; así como lo mencionado en el enfoque reflexivo de la práctica docente propuesto por Gimeno y Pérez (1992), como un medio para la formación y transformación

${ }^{6}$ Cabe señalar que dentro de la presentación de los resultados no se hace una diferenciación entre las aportaciones de los estudiantes con discapacidad y los estudiantes destacados académicamente porque consideramos que las perspectivas de ambos grupos surgen desde estar situados como estudiantes. Si bien cada uno habla desde su centro de enunciación y experiencia, al estar en el aula junto con sus compañeros, tienen una percepción general sobre la práctica de los docentes. 
de la práctica del profesorado. Asimismo, la reflexividad es una capacidad que permite la construcción de la identidad docente; es decir, en el momento en que las y los profesores universitarios reflexionan sobre sus prácticas, sobre la relación con los y las otras, tienen la posibilidad de observarse a sí mismos en esas relaciones y redefinir pensares, sentires y prácticas en esas relaciones (Rodríguez Ugalde, 2016).

\subsubsection{Compromiso con la docencia}

Aquellos profesores que manifiestan atención a su quehacer docente, que atienden a los requerimientos del grupo y los alumnos, que se observan a sí mismos, reflexionan sobre su práctica con el fin de mejorarla, podrían señalarse como profesores que tienen vocación para la docencia y, por lo tanto, muestran un compromiso consigo mismos y con los alumnos. Las respuestas que se incluyeron en esta categoría son: que le guste enseñar, que se encuentre involucrado con la materia, que sea puntual, que pueda adaptarse a las circunstancias que se le presentan, que disfrute al impartir la materia, que tenga vocación. Uno de los docentes dijo:

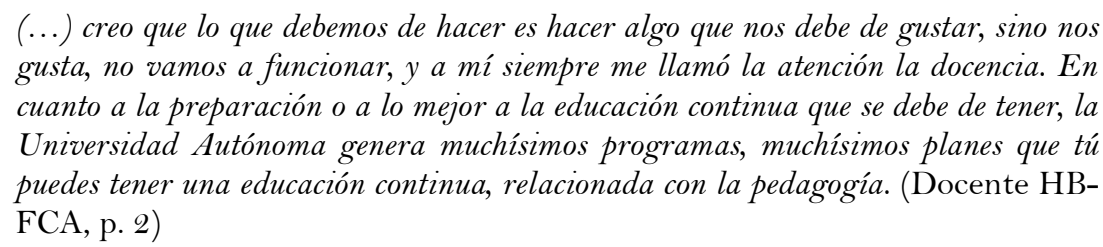
gusta, no vamos a funcionar, y a mí siempre me llamó la atención la docencia. En cuanto a la preparación o a lo mejor a la educación continua que se debe de tener, la Universidad Autónoma genera muchísimos programas, muchísimos planes que tú puedes tener una educación continua, relacionada con la pedagogía. (Docente HBFCA, p. 2)

\subsubsection{Formación docente}

La formación del docente puede verterse en dos orientaciones, una sería el área disciplinar, que compete al contenido académico, el saber del docente sobre la materia; la otra orientación sería la pedagógica, el conocimiento sobre estrategias adecuadas de enseñanza, didáctica, conocer al alumno, identificar cómo se lleva a cabo el aprendizaje. Los alumnos reconocieron que una buena práctica docente es dominar la materia, mostrar conocimiento del tema y que relacionarlo con otras áreas. Los docentes y los alumnos mencionaron que la formación debe de dirigirse tanto al área disciplinar como al área pedagógica:

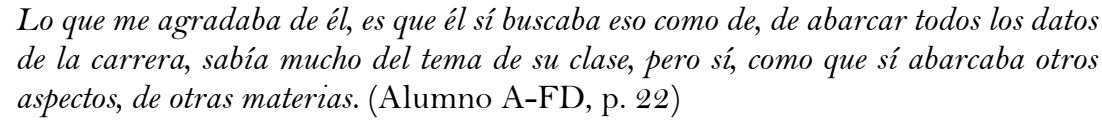

Respecto a ello Porter y Brophy (1988), señalan que un indicador de la buena enseñanza es conocer los contenidos y las estrategias pedagógicas. Por su parte, Blanco (2006) menciona que a los profesores les corresponde practicar enfoques metodológicos que faciliten la diversificación y flexibilidad de la enseñanza.

\subsection{Dimensión social}

\subsubsection{Atención a la diversidad}

En esta categoría se engloban aquellas características actitudinales que los docentes presentan hacia los distintos alumnos que se encuentran en su aula. Fue señalada principalmente por los docentes. Estos docentes mencionaron que les corresponde otorgar un trato igual a los alumnos sin importar sus características. Con esto fue posible apreciar la importancia que otorgaron a la inclusión, considerando que los alumnos son diversos, y respetando esa diversidad en las aulas, atendiendo al derecho que tienen a recibir una educación que respete sus diferencias, lo cual es uno de los principios básicos para las 
buenas prácticas docentes. Tal como señala Blanco (2006), el profesor debe de tener una actitud donde valore y enfatice la diversidad.

Por su parte, los alumnos señalaron el trato igualitario hacia alumnos con y sin discapacidad, la capacidad para atender y ayudar a alumnos con discapacidad, que tengan interés en que todos los alumnos aprendan, y que confíen en la capacidad del alumno y no se basen en los prejuicios sobre sus características físicas:

\begin{abstract}
(...) y es que también creo que los docentes ya deben de ver su profesión de diferente forma, deben [Piensa] de bajarse [Vacila] como que siempre estuvieron en un pedestal, siempre se ven por encima de los estudiantes, entonces ahora debe de estar en un plano de igualdad. Para ser docente se necesita humildad y para hacerlo, cuando haya alumnos con discapacidad, además de la humildad se necesita paciencia y saber adaptarse. Lo que les hace falta a los docentes es saber adaptarse a las circunstancias. (Alumna M-FD, p. 3)
\end{abstract}

Esta característica es mencionada por varios autores como una de las principales que debe de tener un docente, debido a que involucra la disposición hacia el trabajo con el alumnado y la apertura a las diferencias que éste presenta (Colmenero, 2009). En el momento en que el docente tenga la disposición para atender a la diversidad, se encontrará en la posibilidad de observarse a sí mismo en su actuar con el grupo, permitiendo la reflexión sobre su propia práctica y la soltura para realizar trabajo colaborativo con otros docentes, debido a que habrá dejado de ver al alumno desde el paradigma de la deficiencia, y se encontrará en la posibilidad de cambiar la perspectiva hacia el modelo competencial considerando al alumno como capaz de aprender, valorando las diferencias (López Melero, 2001).

\title{
3.2.2. Trabajo colaborativo
}

Los alumnos mencionaron la importancia de compartir con otros compañeros las actividades a realizar, la colaboración para lograr los objetivos planteados. Por su parte, los docentes señalaron el trabajo colaborativo en dos niveles, uno planteado en las actividades planeadas para los alumnos, y otro en la colaboración con otros docentes para reflexionar y mejorar su propia práctica docente.

\footnotetext{
Nos hace interactuar entre nosotros mismos y ahora sí que ayudarnos entre todos, claro que los ejercicios, pues cada quien los hace de forma individual y ya, depende de cada quien, a qué ritmo vayas, pero como es una materia de mucha práctica, no es tanto de hacer un ejercicio entre cuatro personas, sino, más a cuantas dudas, en cuanto a alguna pregunta que se tenga o así. (Alumno R-FCA, p. 7)

Afortunadamente en la Academia que te decía donde yo soy jefe de área, este, la verdad es un grupo de compañeros igual, con muchas inquietudes muy similares, hay mucha coincidencia en ese sentido, muchas inquietudes, porque nuestras materias, sobre todo de las básicas te digo, son muy teóricas, entonces, coincidimos en eso de que ¿̇cómo hacerle para que el alumno no sienta que es demasiada teoría, mucha carga?, entonces ¿cómo hacerle para empezar a llevarlo?, te digo, a desarrollar y compartir estrategias “¿tú cómo le haces, tú qué haces?”, cuando tenemos reuniones se comparte entre los mismos maestros. (Docente AMG-FCA, pp. 12-13)
}

Con lo anterior, se evidencia lo señalado por Gimeno y Pérez (1992) en el enfoque de investigación- acción y formación del profesorado para la comprensión; además de lo dicho por Fernández (2002) respecto al último momento del acto didáctico, el cual es el trabajo colaborativo entre los profesores; así como lo mencionado por Ainscow (2002) sobre el permitir y motivar a los alumnos a trabajar colaborativamente, y el trabajo colaborativo entre los docentes para la mejora del centro. 


\title{
3.2.3. Considerar las características del grupo para la planeación de las clases
}

Un docente que desconoce las características de su grupo plantearía su práctica a "ciegas", teniendo el riesgo de segregar en lugar de incluir. Esta característica la refirieron únicamente los docentes. En ello mencionaron que es importante conocer previamente las características de los alumnos con y sin discapacidad, así como la dinámica del grupo para considerar la forma más adecuada de impartir clases. Esto hace referencia al momento preactivo que menciona Fernández (2002), así como lo señalado por Aguerrondo (1993), sobre conocer las características del sujeto de enseñanza.

\begin{abstract}
Nosotros fuimos sensibilizados (...), no todos los maestros, pero sí a los que en algún momento tuvimos a un alumno con esas características, de que los procesos educativos tienen que ser diseñados, pensando precisamente en todas esas posibilidades de que, cómo interesarlos, cómo lograr que la información llegue por los canales que tengan más desarrollados, entonces eso es lo que hemos estado aprendiendo, cómo diseñar. (Docente GRZ-FD, p. 4)
\end{abstract}

\subsubsection{Dominio de grupo}

El papel del docente ante un grupo es como guía o dirección, para ello se requiere que cuente con capacidad de liderazgo. Este aspecto comprende el saber cómo dirigirse al grupo, y fue una característica de la buena práctica mencionada tanto por docentes como por alumnos.

Principalmente creo que sepan enseñar [titubea] sepan dirigir el grupo, el liderazgo,
hacer amena la clase, porque a veces se da mucho eso en las clases de teoría, que
explican y hasta te da sueño [ríe] (...) y sobre todo en lo que me gusta mucho, en
práctica, es que sean, que sólo te digan cómo hacer, (...) que te digan cómo, más no te
digan, no te lo hagan, porque finalmente, tú eres el que, cuando salgas, el profe no te
va hacer los trabajos afuera. (Alumno R-FCA, p. 11)

\subsection{Dimensión didáctica}

\subsubsection{Planeación de clases}

Dentro de la actividad de un docente se encuentra la determinación del plan de trabajo que se llevará a cabo a lo largo del curso de su materia. Este plan de trabajo involucra desde la exposición clara de los objetivos que pretende, las temáticas a abordar en su curso, las actividades a realizar, los medios, el tiempo de duración y el tipo de evaluación. A pesar de ser una práctica que muchos pueden dar por hecho, no todos los profesores la realizan.

Esta categoría fue mencionada principalmente por los docentes, solamente un alumno lo abordó. Entre las respuestas de los docentes sobre este aspecto es interesante la de un docente que señaló la falta de planeación en sus clases; es decir, que no tiene un documento estructurado para ello. Las respuestas de los demás docentes involucraron el seguimiento de los objetivos planteados en los programas curriculares, otros mencionaron el planeamiento de los objetivos por su propia cuenta, la calendarización de las actividades, dar a conocer a los alumnos cómo será el curso de la materia al inicio del semestre, realizar una planeación didáctica donde se incluyan tiempos, actividades, materiales, objetivos e instrumentos de evaluación.

Al inicio de cada semestre hacemos un calendario, ya una vez que nos dan información sobre el calendario del semestre empezamos nosotros a programar, incluso (...) ya tenemos programadas por semanas cómo vamos a ir teniendo nuestro avance, por ejemplo yo aquí con dos compañeros, la maestra Leticia y el maestro Carlos, tenemos ya organizado todo nuestro curso, por ejemplo este de Bases para el Estudio. Desde el primer día que entramos a clase, con el objetivo por cada unidad que se pretende, pero 
con las actividades, tareas ya asignadas, fechas ya de exámenes, fechas ya programadas de exámenes, (...) eso también nosotros se lo damos a nuestros alumnos, incluso esa información que subimos a la plataforma está en DOQUEOS, para que los alumnos también lo tengan a la mano (...) (Docente AMG-FCA, p. 13)

La mayoría de las personas que se refirieron a la planeación de clases, señalaron esta práctica como algo necesario en la actividad docente, coincidiendo con lo mencionado por Porter y Brophy (1988), Fernández (2002) y Tejedor (2003), respecto a la necesidad de la clarificación de los objetivos a seguir y la planificación de las actividades para una buena práctica.

\title{
3.3.2. Métodos de trabajo
}

Una de las prácticas docentes más importante es el método que siguen los profesores para impartir sus clases. Esta categoría fue mencionada prioritariamente por los profesores; sin embargo, cuatro alumnos con discapacidad también la señalaron como una de las principales características de las buenas prácticas docentes.

Dentro de esta categoría se clasificaron las siguientes respuestas: explica la clase, invita a la metacognición, es descriptivo en las materias, conoce cómo enseñar y transmitir conocimientos, reflexiona sobre los temas que presenta e invita a hacer lo mismo a los alumnos, motiva a los alumnos a analizar, ejemplifica con situaciones de la vida cotidiana, tiene diferentes herramientas de enseñanza.

\begin{abstract}
En este semestre comparto las materias de Taller de Interacción y Aceptación Jurídica y Metodología y Técnicas de la Investigación, pues se les muestra el programa con anticipación, se seleccionan, o en colectivo se les permite seleccionar las actividades y se les encomienda, entregando por escrito un instructivo, donde se les [titubea] señalan los puntos a atender. En este caso, por la naturaleza de la materia de Redacción y Aceptación Jurídica, se les solicita que cuando asisten, cuando asistieron a las conferencias, observen tanto la forma como el tema que se expone, para que tomen nota también del impacto que tiene el expositor en el auditorio, esto es porque en este tercer parcial, los temas que abordamos son la Praxis de la Comunicación Oral que tiene que ver con doble de tiempo, lenguaje, expresión corporal, dominio de vocabulario, entre otros temas. De esa manera, no sólo vemos en clase el concepto de lo que es por ejemplo, la postura que debes tener ante un auditorio, sino que ellos observan y se dan cuenta en la realidad, esa postura que toma el expositor, cuál es el impacto que tiene con la audiencia. Después de que ellos observan y se les pide que tomen nota y hagan un reporte, ahí también tenemos el desarrollo de la expresión escrita, porque no es sólo de ir y presenciar, sino, se les insiste en observar detalles y tomar nota de los detalles; ya en el aula en plenaria se pide que expongan resultados, se les pide que expongan resultados y se haga la puesta en común de a quiénes y cómo observaron. (Docente GRZ-FD, p. 6)
\end{abstract}

Los aspectos señalados por los docentes en cuanto al método hacen referencia a una de las categorías que Blanco (2006) menciona, respecto a la práctica de metodologías que permitan la diversificación y flexibilidad en la enseñanza. Se hace evidente además lo que Tejedor (2003) señaló respecto a que al docente le corresponde considerar las capacidades cognitivas que está desarrollando en los alumnos, así como la presentación de los objetivos de la materia que impartirá, informando a los alumnos las normas de la clase, el tipo de evaluación, y el procedimiento de ésta. Además de lo que Porter y Brophy (1988) señalaron como indicadores de una buena enseñanza: conocer el contenido además de las estrategias pedagógicas, y enseñar a los estudiantes estrategias metacognitivas preparándolos en ellas. 


\title{
3.3.3. Diversidad de actividades
}

En el momento en que el profesor plantea una variedad de opciones para aprender y poner en práctica los aprendizajes adquiridos, los alumnos se encuentran en la posibilidad de vivir más experiencias que les permitan hacer suya la información obtenida. Esta categoría se presentó de forma equitativa entre alumnos con discapacidad y profesores.

Dentro de las respuestas catalogadas en esta categoría, se encuentra el que planifiquen y realicen actividades donde se analicen casos reales, que las clases sean retadoras, visitas de campo, museos, conferencias, diferentes maneras de presentar la información, análisis de películas, lecturas, debates, etc. De este modo, se distingue la posibilidad del alumno para construir su propio conocimiento, retomando lo mencionado por Ainscow, Dyson y Weiner (2013), sobre el planteamiento de actividades que sean retadoras y reales; así como lo señalado en el Diseño Universal del Aprendizaje (CAST, 2008) respecto a los múltiples medios de expresión.

\begin{abstract}
Cuando hablo de las nuevas formas de trabajar es no nada más una sola clase en el salón, hay que ir a conferencias, hay que hacer visitas a empresas, desarrollar otras competencias de los chicos, trabajar en equipo, el liderazgo; sobre todo actividades que a lo mejor en ese momento no generan tanto impacto en los estudiantes, pero cuando ellos salen sí se ven fortalecidos en sus competencias, realmente, yo les incitó, que intentemos trabajar muy dinámico. (Docente HB-FCA, p. 2)
\end{abstract}

\subsubsection{Diversidad de materiales}

Las respuestas de los docentes variaron en cuanto al tipo de materiales que utilizan, algunos mencionaron materiales clásicos como el pintarrón y marcador, mientras que otros muestran apertura a las nuevas tecnologías, considerando distintos medios, por ejemplo, proyecciones con información auditiva y visual. Los alumnos mencionan como herramientas fundamentales aquellas ligadas a las tecnologías, en donde tienen la posibilidad de acceder al tipo de información que, según sus características, les es posible. Enfatizan la relevancia que tiene el que los docentes tengan una actitud de apertura al uso de las nuevas tecnologías.

Siempre procuró que yo le entendiera y siempre procuró tener un apoyo visual. La
mayoría de los maestros dan sus clases verbalmente, algunos utilizan proyección,
algunos pueden utilizar el pizarrón tradicional, pero la maestra siempre tenía un
apoyo visual con los conceptos básicos, y además siempre mostró una gran accesibilidad
para que le pasara mis dudas y siempre me contestaba. Con ella utilicé mucho el correo
electrónico, pero también está la maestra Liliana que me dio clase de informática,
derecho informático, también con ella me ayudó mucho el correo electrónico y como
ella está más familiarizada con estas tecnologías pues ella me lo pidió, también fue
más fácil. (Alumno M-FD, pp. 7-8)

Con lo anterior se muestra lo mencionado por Ainscow, Dyson y Weiner (2013), Blanco (2006), y el Diseño Universal del Aprendizaje (CAST, 2008), respecto a lo oportuno que es contar con múltiples medios de representación de la información para permitir el acceso a la variedad de alumnos que hay en el aula.

\subsubsection{Evaluación}

Es interesante observar cómo los alumnos refirieron la necesidad de una evaluación que permita aplicar lo aprendido más que memorizar y reproducir; además de que el diseño del mismo les permita realizarlo según sus propias características. Respecto a los aspectos tomados en cuenta para la evaluación, los docentes mostraron diferencias, algunos señalaron el considerar sólo el examen, mientras que otros consideran importante 
involucrar algunos otros aspectos como asistencia, participación, trabajos, etc.; hubo quien mencionó que no realiza un examen, sino que evalúa a los alumnos diariamente por medio de sus presentaciones y participación en clase.

Los profesores consideran importante el dar retroalimentación al alumno para que se encuentre al tanto de su desempeño, como lo mencionan Ainscow, Dyson y Weiner (2013) y Porter y Brophy (1988). Hubo maestros que resaltaron la relevancia de la evaluación y retroalimentación de los alumnos hacia ellos para mejorar su práctica docente.

\begin{abstract}
Las evaluaciones, en los comentarios generales, por ejemplo, me empezaba a aparecer que yo era muy elitista, que porque nada más a ciertas personas era con las que yo... entonces, por ejemplo, a mí también esos comentarios me empezaron así como ¿̇por qué los alumnos dicen esto? (...) pero estos mismos comentarios me sirvieron a mí para decir no, el alumno está percibiendo otra cosa, que yo me enfoco nada más con ciertas gentes del grupo, lo clásico, que nada más son unos a los que les pones interés y los demás nos sentimos excluidos. (Docente AMG- FCA, p. 11)
\end{abstract}

\title{
3.4. Dimensión valoral
}

\subsubsection{Motivación del alumno}

El tener interés en la clase es una actitud que permite a los alumnos acceder a la información y al aprendizaje. Tanto alumnos como profesores identificaron que una de las características de las buenas prácticas docentes es la actitud del docente hacia la participación e involucramiento del alumnado en la dinámica misma de la clase. Dentro de las respuestas que caracterizan a esta categoría se encuentran que el docente realice preguntas a los alumnos en el momento que se encuentra exponiendo el tema, que motive la autonomía de los alumnos, los incite a ser analíticos, denote el interés de los alumnos, así como la iniciativa del estudiante, permitiendo que él mismo identifique cómo conducirse para resolver un problema o desarrollar un proyecto; que permita la participación del alumno, no limite al alumno con discapacidad, que se encuentre al tanto del progreso de los alumnos y esté involucrado con su desempeño.

Incluso a veces, por ejemplo, ahorita en últimos semestres, la mayoría de las
herramientas, los modelos, ellos ya los vieron en otros cursos, entonces en lugar de yo
llegar y dárselo teóricamente porque ya teóricamente ellos ya lo vieron, incluso es
"bueno a ver tú me lo vas a explicar, pero me lo vas a explicar a través de un ejemplo,
de una empresa que hayas utilizado vas a decirles a los compañeros cómo fue que ellos
lo utilizaron", y obviamente de ahí ¿qué estamos haciendo?, estamos reforzando lo que
tú ya viste teóricamente. (Docente AMG-FCA, p. 8)

Esta categoría conlleva lo que Tejedor (2003) señala, al mencionar que el interés y rendimiento de los alumnos aumenta si el docente se muestra accesible a ellos, los asesora y orienta, es entusiasta el exponer el tema y estimula su participación.

\subsubsection{Clima de confianza para facilitar la participación}

Para que el proceso de enseñanza aprendizaje involucre a los alumnos activamente, requiere de propiciar una dinámica en el aula adecuada para ello. En el momento en que el profesor se muestra accesible a los alumnos, los orienta, los escucha, tiene apertura a los comentarios, respeta los distintos puntos de vista y los alumnos realizan lo mismo hacia él, se puede decir que existe un clima adecuado que facilita la participación del alumnado. Como señala Blanco (2006), al docente le corresponde generar un buen clima afectivo y emocional en el aula y en la escuela. De igual forma, Ainscow, Dyson y Weiner (2013) menciona que un docente con buena práctica otorga reconocimiento a los alumnos sobre sus esfuerzos y logros. Tejedor (2003) identifica que uno de los comportamientos de un 
docente para una enseñanza de calidad es encontrarse accesible a los alumnos, orientarlos y asesorarlos.

Tanto alumnos como profesores coinciden en la necesidad de que el docente se encuentre disponible para acercarse a expresar sus dudas. Se requiere que exista una comunicación constante con el alumno, en donde el docente no se encuentre en un pedestal, sino que muestre comprensión a las vivencias que ellos tienen.

Que hacer una, [piensa] algunas rupturas con el discurso, con temas, con ejemplificarles con temas más cercanos a su cotidianidad y que vengan también de mi cotidianidad, entonces eso los hace ver como que el profesor no es el que, no es tan lejano... tonterías si quieres verlas así, como de pronto, estoy explicando filosofía y te uso un capitulo del The Big Bang Theory o de un capitulo de los Simpson, que a mi me gustan y que ellos conocen. (Docente AR-FD, p.11)

\section{Conclusiones}

A partir de los resultados mostrados en el análisis de las prácticas docentes realizado en la FCA y la FD, es posible notar que los alumnos y los docentes entrevistados redundan en aquello que consideran como características de las buenas prácticas. Fue interesante el hecho de que los resultados fueron muy similares a los estudios recientes sobre el tema realizados por Fernández, Maiques y Ábalos (2012) y Álvarez, Porta y Sarasa (2011).

Conocer aquello que tanto alumnos como profesores refieren como buenas prácticas docentes, permite tener una guía sobre lo que se requiere fortalecer en la formación docente para atender a la diversidad en el nivel de educación superior, específicamente en la FCA, y darnos cuenta que es mucho lo que se tiene que realizar. Desde aspectos básicos que se dan por hecho en la planeación de las clases, como actitudes hacia la diversidad, así como la reflexión y el trabajo colaborativo.

Con base en el análisis, podemos señalar que la práctica docente se conforma de la dinámica de elementos que corresponden a distintos niveles del sistema social; es una confluencia entre lo macro y lo micro. Lejos de determinarse como una acción técnica, involucra las concepciones en conjunto con los comportamientos, en la bidimensionalidad ya referida por Zabalza (2012). En ocasiones es difícil discernir los elementos que corresponden a cada dimensión, ya que en el mundo de lo relacional las fronteras son sutiles. Sintetizamos así que la práctica docente es un medio a través del cual se materializa la identidad del docente.

Es pertinente el trabajo con el resto del cuerpo docente de estas facultades, debido a que son centros universitarios donde se encuentran alumnos con diferencias evidentes como la discapacidad (López, Méndez y Mendoza, 2014; Vázquez, Méndez y Mendoza, 2015), que podrían encontrarse en riesgo de exclusión. Sin embargo, no son los únicos a quienes beneficiaría la inclusión por parte de los profesores, sino que un trabajo de intervención orientado a la inclusión beneficiará a todos los alumnos inscritos. Además, en el momento en que el resto de los docentes tengan apertura hacia el trabajo con todos sus alumnos, desarrollarán habilidades para enfrentarse a otros retos en el aula.

La formación, tanto en el área académica como pedagógica, por parte de los docentes de educación superior es necesaria para permitir el acceso a la participación y al aprendizaje a todos los alumnos. Es evidente que la UASLP ofrece cursos en donde los profesores pueden prepararse académicamente $\mathrm{y}$, de manera paralela, se han desarrollado otras 
propuestas de formación dirigidas al área pedagógica, de las cuales algunas se ofrecen en el Instituto de Ciencias Educativas de la UASLP. Sin embargo, la audiencia es baja y se imparten en contextos que son completamente ajenos al área de labor diaria de estos docentes.

Es necesario involucrar a los docentes como actores principales de su propia formación en el desarrollo de buenas prácticas docentes, y el enfoque de investigación- acción es acorde a ello. Se requiere de la formación docente en trabajo colaborativo con otros docentes, que permita la reflexión en conjunto, en el ambiente mismo donde desempeñan su función y con las dificultades y características que le son propias a éste. Las características de las prácticas docentes definidas como buenas en este diagnóstico son pautas iniciales para orientar un proceso de formación docente dentro de la FCA bajo el enfoque de investigación-acción.

Cabe decir que dentro de las limitaciones del estudio se encuentran: el pequeño número de participantes; la falta de otras fuentes de información que sirvieran como evidencia, tales como la observación directa de la práctica docente en el aula; los resultados no pueden definirse como aplicables para el universo de las universidades, en razón de lo señalado en la definición de las buenas prácticas docentes: son buenas acorde con los contextos.

\section{Referencias}

Aguerrondo, I. (1993). La calidad de la educación: Ejes para su definición y evaluación. Revista Interamericana de Desarrollo Educativo, 6(3), 13-28.

Aguilar, S. y Barroso, J. (2015). La triangulación de datos como estrategia en investigación educativa. Revista de Medios y Educación, 47, 77-88. https://doi.org/10.12795/pixelbit.2015.147.05

Ainscow, M. (2002). Rutas para el desarrollo de prácticas inclusivas en los sistemas educativos. Revista de Educación, 327, 69-82.

Ainscow, M., Dyson, A. y Weiner, S. (2013). De la exclusión a la inclusión. Una revisión literaria internacional en camino para responder a los estudiantes con necesidades educativas en las escuelas. Clave Pedagógica, 13, 13-30.

Álvarez, Z., Porta, L. y Sarasa M. (2011). Buenas prácticas docentes en la formación del profesorado: Relatos y modelos entramados. Profesorado: Revista de Currículum y Formación del Profesorado, 15(1), 229-240.

Amaro, M. C., Méndez, J. M. y Mendoza, F. (2014). Un estudio de las características profesionales del docente universitario para atender a la diversidad. Revista Latinoamericana de Inclusión Educativa, 8(2), 199-216.

Bertely, M. (2000). Conociendo nuestras escuelas. Un acercamiento etnográfico a la cultura escolar. Ciudad de México: Paidós.

Blanco, R. (2006). La equidad y la inclusión social: Uno de los desafíos de la educación y la escuela hoy. REICE. Revista Iberoamericana sobre Calidad, Eficacia y Cambio en Educación, 4(3), 1-15.

Booth, T. y Ainscow, M. (2000). Índice de inclusión: Desarrollando el aprendizaje y la participación en las escuelas. Bristol: UNESCO.

Camacho, M. A. (2013). Prácticas de tutoría para la atención a la diversidad. Tesis de maestría. Universidad Autónoma de San Luis Potosí, México.

CAST (2008). Universal design for learning guidelines version 1.O. Wakefield, MA: Author. 
Colmenero, M. (2009). Influencia y repercusión de la experiencia como docente en la atención a la diversidad. Su incidencia en la formación. Revista de Educación Inclusiva, 2(3), 71-82.

Contreras, J. (2002). Educar la mirada y el oído. Percibir la singularidad y también las posibilidades. Cuadernos de Pedagogía, 311, 61-65.

Fernández, A. (2002). Ideas para seguir reflexionando sobre educación. Barcelona: Universitat Autónoma de Barcelona.

Fernández, A., Maiques, J. y Ábalos, A. (2012). Las buenas prácticas docentes de los profesores universitarios: Estudio de casos. Revista de docencia universitaria, 10(1), 43-66. https://doi.org/10.4995/redu.2012.6121

Fierro, C., Fortoul, B. y Rosas, L. (1999). Transformando la práctica docente. Una propuesta basada en la investigación Acción. Ciudad de México: Paidós.

García, N. (2004). Diferentes, desiguales y desconectados. Mapas de la interculturalidad. Barcelona: Gedisa.

Gentili, P. (2001). La exclusión y la escuela: el apartheid educativo como política de ocultamiento. Política Educativa, 5, 4-11.

Gimeno, J. y Pérez, A. (1992). Comprender y transformar la enseñanza. Madrid: Morata.

Gómez, I. (2008). La interculturalidad en las aulas. Revista Digital Enfoques Educativos, 13, 43-48.

Lloret, C. (1994). Nos- altres. Revista Clic, 5(2), 35-38.

López Melero, M. (2001). La cultura de la diversidad o el elogio de la diferencia y la lucha contra las desigualdades. En A. Sipán (Coord.), Educar para la diversidad en el siglo XXI, (pp. 31-65). Zaragoza: Mira.

López, C. A, Méndez, J. M. y Mendoza, F. (2014) Construyendo políticas de equidad en los procesos de admisión. El caso de la Universidad Autónoma de San Luis Potosí. En J. Gairín, G. Palmeros y A. Barrales (Coords.), Universidad y colectivos vulnerables (pp. 676-689). Ciudad de México: Accedes.

Maya, C. (2016). Formación docente en tutoría inclusiva universitaria: Propuesta educativa para atender a la diversidad estudiantil. Tesis de maestría. Universidad Autónoma de San Luis Potosí, México.

Maya, C., Méndez, J. M. y Mendoza, F. (2017). Atención a la diversidad estudiantil: Retos en el contexto universitario mexicano. Revista de Investigación en Educación, 15(1), 62-74.

Méndez, J. y Mendoza, F. (2007, noviembre). Actitudes hacia los alumnos con capacidades diferentes en la Universidad Autónoma de San Luis Potosí. Ponencia presentada en el IX Congreso Mexicano de Investigación Educativa. Mérida, México.

Nussbaum, M. (2012). Crear capacidades: propuesta para el desarrollo humano. Barcelona: Paidós Ibérica.

Ordine, N. (2013). La utilidad de lo inútil. Manifiesto. Barcelona: Acantilado.

Porter, A. y Brophy, J. (1988). Synthesis of research on good teaching: Insights from the work of the institute for research on teaching. Revista Educational Leadership, 45, 74-85.

Rodríguez Ugalde, D. C. (2014). Formación del profesorado en prácticas inclusivas en la FCA de la UASLP. Tesis de maestría. Universidad Autónoma de San Luis Potosí, México.

Rodríguez Ugalde, D. C. (2016). Ser docente universitario para la diversidad: Un proceso de reflexión y re-construcción. En D. Solís Domínguez (Coord.), Perspectivas socioculturales sobre exclusión y violencia en la educación (pp. 103-119). Ciudad de México: Fontamara. 
Santos, B. S. (2015). La universidad en el siglo XXI. Ciudad de México: Siglo XXI.

Santos Guerra, M. (2006). La estrategia del caballo y otras fábulas para trabajar en el aula. Buenos Aires: Homo Sapiens.

Tejedor, F. (2003). Un modelo de evaluación del profesorado universitario. Revista de Investigación Educativa, 21(1), 157-182.

Vázquez M. G., Méndez, J. M. y Mendoza. F. (2015). Educación inclusiva y aprendizaje colaborativo en el aula: un estudio de la práctica docente universitaria. Revista de Educación Inclusiva 8(3), 171-187.

Zabalza, M. A. (2012). El estudio de las "buenas prácticas" docentes en la enseñanza universitaria. Revista de Docencia Universitaria, 1O(1), 17-42. https://doi.org/10.4995/redu.2012.6120

\section{Breve CV de los autores}

\section{Diana Cecilia Rodríguez Ugalde}

Profesora de la Universidad Pedagógica Nacional Unidad 241 (México); profesora invitada en la Universidad Autónoma de Querétaro. Estudiante del Doctorado en Estudios Latinoamericanos en Territorio, Sociedad y Cultura, en la Facultad de Ciencias Sociales y Humanidades de la UASLP. Maestra en Psicología con orientación en el área de Educación y Sociedad por la misma institución. Licenciada en Psicología por la UASLP. Áreas de formación: Psicoterapia con enfoque sistémico, Psicología educativa, Antropología y Sociología de la Educación. Líneas de investigación/intervención: Formación del profesorado, Identidad del profesorado indígena, Educación inclusiva y Educación para/por los pueblos originarios, Perspectivas decoloniales. ORCID ID: 0000-0003-3945-4600.Email: psic.diana.rdz.ugalde@hotmail.com

\section{Fernando Mendoza Saucedo}

Es profesor investigador de tiempo completo en la Universidad Autónoma de San Luis Potosí, (México), tiene estudios de Licenciatura en Psicología y Maestría en Investigación Educativa y pertenece al cuerpo académico en consolidación: "Psicología y Educación" reconocido por PRODEP con clave UASLP-CA-195. Sus líneas de investigación son la formación docente, escuela de la diversidad, orientación y tutoría. Imparte docencia en licenciatura y posgrado en la Facultad de Psicología y otras instituciones educativas de carácter público y privado. Cuenta con perfil deseable PRODEP 2015-2108. Es miembro fundador del Centro de Investigación, Orientación y Apoyo a la Inclusión (CIOAI) y director del Instituto de Ciencias Educativas de la UASLP. ORCID ID: 0000-0002-0200-9899. Email: fmendoza@uaslp.mx

\section{Juana María Méndez Pineda}

Es profesora investigadora de tiempo completo en la Universidad Autónoma de San Luis Potosí (UASLP) (México). Es Licenciada en Psicología por la UASLP, Maestra en Investigación Educativa por la Universidad de Guanajuato, Doctora en Educación por la Universidad Iberoamericana Plantel Golfo Centro y tiene estudios de especialización en Formación de Educadores de Adultos por la Unidad 241 de la Universidad Pedagógica Nacional. Coordina el Centro de Investigación, Orientación y Apoyo a la inclusión del Instituto de Ciencias Educativas. Es líder del Cuerpo Académico "Psicología y Educación”; cultiva las líneas de investigación "Educación y diversidad" y "Formación docente". Es miembro del Sistema Nacional de Investigadores de CONACYT. ORCID ID: 0000-0001-5428-8589. Email: jpineda@psicologia.uaslp.mx 